Fecha de recepción: febrero 2019 Fecha de aceptación: marzo 2019 Versión final: mayo 2019

\section{La insurrección de los saberes sometidos: una interpretación del film Talentos Ocultos desde la antropología de la ciencia}

Adriana Alejandrina Stagnaro *

Resumen: El propósito del presente trabajo es interpretar a las prácticas y discursos sociales surgidos del film Talentos ocultos dirigida por Theodore Melfi (2016), desde el enfoque de la antropología de la ciencia sobre la relación entre ciencia, género y poder.

La película, un largometraje biográfico basado en el libro de no ficción Hidden Figures de Margot Lee Shetterly, rescata las trayectorias personales y científicas de Katherine Johnson, Dorothy Vaughn y Mary Jackson como calculistas afroamericanas de la NASA, invisibilizadas, desfavorecidas y segregadas en una época teñida por la lucha por el reconocimiento de los derechos civiles y la inclusión social. El film reaviva y explora el drama humano expresado por su doble condición de mujeres y afrodescendientes en una comunidad científica preponderantemente masculina interesada en revertir el avance soviético en el dominio del espacio y desarrollar competitivamente la carrera espacial de los Estados Unidos.

El material aportado por el film, muy concordante con el libro en que se basa, permite analizar no sólo el rol de la mujer como sujeto hacedor de la ciencia, sino ir más allá en la comprensión de sus prácticas y representaciones científicas, analizando sus avances y límites en la producción del conocimiento, sus especificidades aportadas en los contenidos obtenidos y el tipo de saberes situados socialmente. En tal sentido, la antropología de la ciencia, en especial sus pioneras provenientes del campo de los estudios feministas y culturales, aporta un bagaje específico de tratamiento teórico que reconocen en gran parte aunque no unánimemente los novedosos conceptos aportados por Foucault (1976) sobre el resurgimiento de los saberes soterrados por una ciencia detentada por unos pocos.

Palabras clave: Ciencia - género - poder - cultura - antropología

[Resúmenes en inglés y portugués en las páginas 119 - 120]

${ }^{(*)}$ Antropóloga. Doctora en Antropología Social por la Universidad de Buenos Aires. Profesora de la cátedra de Epistemología y Métodos de la Investigación Social en la Carrera de Ciencias Antropológicas de la Facultad de Filosofía y Letras y de Epistemología de las Ciencias Sociales en la Carrera de Trabajo Social, Facultad de Ciencias Sociales de la UBA. Como investigadora del Instituto de Ciencias Antropológicas de la Facultad de Filosofía y Letras fue una de las iniciadoras de la Antropología de la Ciencia y la Tecnología, especialidad sobre la que ha escrito artículos y capítulos de libros y sobre la que ha orientado su investigación, siendo su última publicación "Ciencia a pulmón. Etnografías de laboratorios argentinos de biotecnología". 2015. Buenos Aires, Ciccus. Integra el Proyecto UBACYT 2018- 2020/ 20020170100593BA Grupos consolidados. Tema: Co-producción 
de conocimiento: nuevos formatos asociativos y materialidad de la creatividad científica. Facultad de Filosofía y Letras. Directora: Dra.Cecilia Hidalgo.

- El presente trabajo se realizó con el apoyo del UBACYT No 20020170100593BA 20182021 del proyecto "Co-producción de conocimiento: nuevos formatos asociativos y materialidad de la creatividad científica" dirigido por la Dra.Cecilia Hidalgo

\section{Introducción}

Las resistencias no dependen de algunos principios heterogéneos; más no por eso son engaño o promesa necesariamente frustrada. Constituyen el otro término en las relaciones de poder; en ellas se inscriben como el irreductible elemento enfrentador. Las resistencias también, pues, están distribuidas de manera irregular: los puntos, los nudos, los focos de resistencia se hallan diseminados con más o menos densidad en el tiempo y en el espacio, llevando a lo alto a veces a grupos o individuos de manera definitiva, encendiendo algunos puntos del cuerpo, ciertos momentos de la vida, determinados tipos de comportamiento. (Foucault, 2008, p.92)

\section{Saberes sometidos}

Es casi ineludible, en una primera e ingenua interpretación del discurso cinematográfico bajo análisis, traer el concepto de Foucault los retornos del saber para referirse a lo que él denomina la insurrección de los saberes sometidos para ponerlo en juego, aunque más no sea que como un ejercicio de simple analogía o correspondencia semántica con el título Talentos Ocultos.

Bajo el enunciado saberes sometidos Foucault (1992, p.130) distingue dos tipos de hechos diferentes. Por un lado, se refiere a los contenidos históricos que se han mantenido hundidos y enterrados en el interior de sistemas funcionales o formales permitiendo reencontrar la ruptura, los enfrentamientos y la lucha que aquéllos tienen por fin ocultar. Esos bloques de saberes históricos soterrados reaparecen por medio de la crítica basada en la erudición. Por el otro, entiende por saberes sometidos algo paradojalmente diferente: un conjunto de saberes considerados como incompetentes, imperfectos, ingenuos, inferiores al conocimiento científico saberes que denomina saberes de la gente, los cuales no han llegado a constituir un saber común, sino que más bien se tornan saberes específicos, locales, regionales y diferenciales. Para Foucault la emergencia o irrupción de estos saberes de la gente que deben su fuerza a la dureza que lo opone a lo que le rodea es el locus donde opera la crítica.

Ambos tipos de saberes, los eruditos y los descalificados conservan y retienen la memoria de los enfrentamientos, el saber histórico de la lucha. Sus tensiones, relaciones de fuerza y disputas remiten al tema del poder, a la pregunta de cómo se ejerce ese poder, cómo se inscriben los sucesos en los cuerpos impregnados de historia y en qué prácticas sociales se despliegan las relaciones de fuerza. 
A fin de articular estas palabras con el relato de la película y sus tópicos, volvamos a ella. La primera escena (intriga de predestinación) remite a un momento crucial, cuando en los años 20' la pequeña y lúcida Katherine Johnson es beneficiada por una beca para continuar con sus estudios de las matemáticas, que obliga a sus padres a trasladarse con ella a otra ciudad y cambiar sus planes. Primera ruptura de la vida cotidiana que permite el ingreso al universo de las matemáticas. Luego vendrán otros, el ingreso a la Facultad para afroamericanos y finalmente su incorporación a un laboratorio de la NASA, momento este último donde se centra la trama y la mirada cinematográfica. En este último ámbito Katherine logró realizar los cálculos exitosos de las trayectorias de lanzamiento y aterrizaje que hicieron posible la puesta en órbita del astronauta John Glenn en 1961, el vuelo del Proyecto Mercurio y la llegada a la luna del Apolo 11 en 1969 por parte de los Estados Unidos. Participa en sus primeros tiempos con sus colegas Dorothy Vaughan y Mary Jackson en las tareas del área segregada de calculistas negras, llamadas a participar en el proyecto tendiente a igualar y superar los avances espaciales de la entonces Unión Soviética. Conjuntamente se enfrentan con valentía a una institución que las destrata y pretende ocultarlas, pero que no obstante las necesita. Sus luchas cotidianas para ser reconocidas intelectual y socialmente en un ambiente claramente segregado donde se les imponen fuertes desafíos, se articulan con el escenario histórico marcado por el accionar de los movimientos por los derechos civiles que bregan por la inclusión y ascenso social de la población negra. La enérgica defensa de sus derechos abarca tanto la demanda de ser copartícipes en los momentos científica y técnicamente decisivos, de los cuales eran excluidas, la ganada autoría de los informes y papers que tradicionalmente eran firmados y apropiados por parte de sus colegas blancos hasta el extremo de poder usar los mismos espacios, haciendo desaparecer los denigrantes carteles " black people" de mesas y baños. Aquí, la recurrente imagen del correr desesperado de Katherine Johnson para alcanzar los baños destinados a las calculistas negras, a gran distancia de su laboratorio, ilumina el dispositivo de poder tendiente a establecer la discriminación en forma natural, "impensada" por los arquitectos del área blanca y menos por sus ocupantes. Sólo el grito desesperado de Katherine lo podrá hacer palpable. Sus compañeras de cómputos y amigas en la vida externa al ámbito laboral también tienen sus propias luchas: demandar igual salario entre científicas negras y blancas, mayor reconocimiento de sus capacidades informáticas e ingenieriles, posibilidad de ascensos en las jerarquías de la institución, reclamo por las condiciones de trabajo. Todas deberán enfrentar con diversas estrategias los prejuicios raciales, las trampas legales y miles de otros obstáculos diarios que les impiden su realización profesional. Ninguna de ellas es militante por los derechos civiles, aunque una de ellas está casada con un activista que le hace ver la sobreexplotación a la que la someten. Pero la palabra de Martin Luther King está constantemente detrás de la pantalla y del relato, como increpación y como aliento.

Todas las prácticas y discursos de resistencia que impregnan el relato pueden ser interpretadas en relación al concepto foucaultiano de insurrección de los saberes sometidos en dos dimensiones yuxtapuestas: la de la resistencia y lucha promovidas por los saberes eruditos, y la emergencia y aparición de los saberes descalificados, para poner en evidencia las relaciones de poder existentes en el momento histórico tratado. 
Así, la participación plena de estas científicas dentro del fuerte amurallado de los saberes eruditos y legitimados, gracias a sus conocimientos de los contenidos propiamente cognitivos y técnicos que posibilitan su entrada en el edén matemático, no autorizan sin embargo su legitimación automática como científicas dentro de una comunidad científica homogéneamente blanca. Ahí operan entonces, las condiciones históricas opacadas por el poder y fuerza de la institución que rechaza la heterogeneidad comunitaria y la inclusión de los cerebros negros como partícipes iguales. Ellas tienen y han tenido en sus trayectorias acceso al conocimiento, pero ese conocimiento no genera efectos de poder en la comunidad científica para ser reconocidas como tales. Están destinadas a un papel subalterno.

Por otra parte, como portadoras corpóreas de las luchas sociales de los afroamericanos por sus derechos, son portadoras también de saberes sometidos, fraguados en la confrontación histórica, e impulsados a irrumpir en cualquier contexto, para lograr su visibilidad y calificación. Estos saberes de la gente, de la gente de color en un país cruelmente discriminatorio están íntimamente imbricados con las condiciones de producción de los saberes eruditos que, una vez legitimados, borran todos los elementos impuros, que estuvieron presentes en el proceso de su producción. Contra este efecto de poder que ha logrado suprimir los condicionamientos sociales, se rebelan los cuerpos y mentes de estas científicas.

\section{Ciencia, tecnología y género}

Los estudios de la ciencia y la tecnología desde la perspectiva de género constituyen hoy un campo de investigación muy fértil que alberga múltiples perspectivas teóricas y diversas disciplinas. No obstante su heterogeneidad, todos comparten un objetivo central: el rechazo al sexismo y al androcentrismo expresados en la práctica científica.

Todos estos abordajes entre los cuales encontramos a la antropología de la ciencia y la tecnología reconocen como antecedente común al movimiento feminista, en especial al movimiento de liberación de la mujer de los años 60 y 70 (Franklin, 1995; Fischer, 2007). La discusión sobre el lugar ocupado por las mujeres en el desarrollo de la ciencia y la tecnología se centra en la conciencia histórica de la diferencia, de su número reducido y escasa producción y de los obstáculos puestos al acceso de las mujeres al campo de la ciencia. Los trabajos históricos, sociológicos y antropológicos que se dan por objetivo recuperar para la historia de la ciencia las figuras femeninas silenciadas y relegadas, son un aporte central para el análisis de Talentos Ocultos, ya que surge claramente que la narrativa cinematográfica, al igual que el libro de base, tienen como objetivo principal denunciar la invisibilización y exclusión, reflotando la vida y saberes de las tres científicas, desconocidas hasta ese momento por el mundo.

Una posible lectura crítica de la película debe resaltar la necesidad de incorporar el género como elemento importante para el análisis de la cultura científico-tecnológica, la cual ha sido caracterizada como sexista, basada en normas y valores de control, dominación y competencia ligados al poder masculino.

En tal sentido, el diálogo entre Mary Jackson y su jefe Zielinsky, asombrado de que ella hubiese dado con la causa del porqué se desprendían pedazos de metales de una parte del cohete, expresan este carácter reforzado por el racismo: 
-¡Mary! Una persona con la mente de un ingeniero, debería de ser un ingeniero. No puedes ser una computadora (calculista) el resto de tu vida.

-Señor Zielinski, soy una mujer negra. ¡No voy a buscar lo imposible!

-Y yo soy un judío polaco cuyos padres murieron en un campo de prisioneros nazis. Ahora estoy de pie bajo una nave espacial que va a llevar a un astronauta a las estrellas. Creo que podemos decir, que estamos viviendo lo imposible. Déjame preguntarte: si fueras un hombre blanco, ¿querrías ser un ingeniero?

- No tendría que hacerlo. Ya sería uno.

También surge el problema de la jerarquización de los saberes. Ser calculista implicaba no ser científicas plenas, sino trabajadoras en una tarea rutinaria y poco reconocida, en el caso frente al saber ingenieril, tradicionalmente vinculado a la capacidad intelectual masculina. Cuando Mary decide estudiar ingeniería, deberá llegar a una corte judicial para que la dejen ingresar en los cursos.

Perez Sedeño (1998) sostiene que una mirada de género debe preguntarse por el tipo de consecuencias que tiene la exclusión de las mujeres para los contenidos y las prácticas científico-tecnológicas. "Su interés y preocupación principal, por tanto, es la de documentar la ausencia y presencia de la mujer en la historia del desarrollo científico-tecnológico, explicar esta situación y proponer estrategias institucionales y educativas para una incorporación más plena de la mujer en estos campos" (Pérez Sedeño, 1998, p. 33)

Tanto el filme como el libro analizados, implican una reescritura de la historia y una apelación a la memoria para recuperar la omisión de mujeres y de sus trayectorias en ámbitos femeninos cuyas prácticas han sido consideradas secundarias en el plano cognitivo. Pese a haber hecho nuevos aportes de saberes tecno-científicos han sido silenciadas por la historia tradicional, debido a distintos tipos de soslayos, ya sean de género, raza o clase social. Así ambas obras contribuyen con una perspectiva original que desmorona las concepciones estrechas de la historia de la ciencia hagiográficas, tendientes a reconstruir la vida de los héroes y personajes de las más importantes disciplinas o de grandes proyectos científicos y tecnológicos -como es el caso-, en base a las trayectorias abnegadas de grandes personajes y de sus teorías o prácticas exitosas, dejando de lado otras actividades y saberes en absoluto marginales al desarrollo de la ciencia.

Posteriormente, la historia de las científicas olvidadas y de sus logros escrita por historiadoras de la ciencia, feministas o concientizadas con la exclusión de la mujer ha producido admirables resultados.

Una de las figuras científicas estudiadas desde esta perspectiva es la de Rosalind Franklin. Sus originales y tempranas fotografías por difracción de rayos X fueron esenciales para que Watson y Crick pudieran proponer el modelo de doble hélice del ADN en 1953 con el que ganarían el Premio Nobel en 1962. El aporte de Rosalind Franklin no sólo fue ocultado, sino que llegó a ser minimizado en la famosa autobiografía de Watson (1968), sin reconocer su importancia.

Esta situación cambia cuando aparece la biografía de Anne Sayre (1975), donde se relata otra historia, presentando la difícil posición académica de la científica fallecida muy joven de familia judía en una institución masculina y anglicana muy reconocida, el King's College de Londres (Alic, 1986; Pérez Sedeño, 1994, González García y Pérez Sedeño, 2002) 
Otros enfoques de historiadoras de la ciencia -en línea con el de Talentos Ocultos-, van a indagar el contexto familiar, social y económico dentro de los cuales se desarrollan y brillan estas mujeres no sólo por sus genialidades sino por sus apuestas y luchas en un campo adverso y excluyente. El resultado es un conocimiento situado (Haraway, 1991), condicionado por el sujeto cognoscente y su situación particular (espacio-temporal, histórica, social y cultural) y la necesaria articulación entre conocimiento y poder.

\section{Perspectivas antropológicas de la ciencia, tecnología y género}

En 1980 y 1990 el diálogo establecido por la antropología y los estudios feministas, culturales, postcoloniales y de los medios de comunicación produjo como resultado las etnografías de la ciencia y la tecnología que comenzaron con los trabajos pioneros de Sharon Traweek (1988), Emily Martin (1987) y Donna Haraway (1989), Sheila Jasanoff (1990), Marilyn Strathern (1992) -entre otras- quienes comenzaron a indagar diversos temas vinculados a la mujer en la ciencia. Provenían del feminismo y aportaron sus conceptualizaciones para estudiar las comunidades científicas desde dentro, analizar sus prácticas y discursos y ver las diferentes formas de socialización para llegar a ser científicas en contraste con las trayectorias masculinas.

Por ejemplo, Sharon Traweek (1988) enfocó el estudio de las comunidades de científicos en física de partículas, llevando adelante estudios comparativos de la comunidad de físicos en Estados Unidos y Japón, describiendo la manera diferencial de formación profesional, de hacer ciencia, de las prácticas en el laboratorio, según fuesen dirigidos por hombres y mujeres y las diversidades culturales e históricas de cada comunidad.

Donna Haraway (1989) presenta desde una mirada culturalista y feminista la forma entremezclada en que se dan las prácticas, las creencias folk o tradicionales y las ansiedades de las investigaciones con las afirmaciones "científicas" de primatologistas y sociobiologistas en las comunidades científicas que estudian simios y primates. Así analizó detalladamente las publicaciones, los papers, la correspondencia y la historia de las expediciones e instituciones de estudio de los primates, revelando la construcción histórica de los pedigrees y la naturalización de la raza, sexo y clase. Siempre buscando y descubriendo las raíces de género de la ciencia entendida como cultura, es decir enfrascada en el medio cultural. La antropóloga Emily Martin (1987) junto con su grupo de investigación realizó 200 entrevistas a personas en distintos barrios de Baltimore para comprender cómo la gente común entendía el sistema inmunológico y cómo sus interpretaciones conforman su pensamiento acerca de la salud, enfermedad y estado físico. A través del análisis de la cultura popular de los años 1940 y 1950, Martin muestra que el cuerpo era pensado como una fortaleza con sus defensas prioritariamente en la superficie. La investigación mostró la representación de que el cuerpo estaba defendido internamente por un complejo sistema inmune para responder a los cambios bruscos. Esa adaptabilidad o flexibilidad es considerada un atributo deseable no sólo para el sistema inmunológico sino también para individuos y organizaciones y su falta genera menos capacidad de supervivencia tanto biológica como económicamente. En tal sentido advierte los peligros de esa mirada y promueve 
por el contrario valores de estabilidad y seguridad. Y en general trata de mostrar cómo las ideas científicas operan en el mundo real.

Finalmente como corolario de este paneo presentamos el aporte de Marilyn Strathern (1992) cuyos trabajos ejemplifican el paso de género-a-ciencia, afirmando que los estudios de género y parentesco tienen un objetivo en común: criticar el estatus a priori concedido a los hechos biológicos, demostrando por el contrario el carácter híbrido del parentesco, tomando como base de estudio los debates sobre la paternidad, la procreación y el parentesco en el contexto de las nuevas tecnologías reproductivas. Estos cambios conceptuales y culturales interpelan a las "neutrales" conceptualizaciones científicas de modo esencial. Todas estas investigaciones enriquecen el estudio de la cultura científica tradicional con su mirada feminista y posibilitan un cambio en la perspectiva más masculina de los estudios de laboratorio y comunidades científicas, y aportan también herramientas conceptuales novedosas para transitar desde los primeros estudios de la mujer en la ciencia hacia una interrogación de qué elementos conforman la ciencia y cómo sus propios conceptos se hallan permeados por rasgos de jerarquía, género y dominación.

En nuestra reflexión tales aportes constituyen una plataforma de partida para repensar la narrativa de Talentos Ocultos y repreguntarse qué clase de ciencia se hacía en ese momento y cuales eran los elementos heterogéneos que conformaban las conceptualizaciones del núcleo duro de su pensamiento. ¿Habrá habido alguna contribución distintiva de las rutinarias y desjerarquizadas calculistas a las teorías científicas en cuestión? La escena donde Katherine resuelve un problema matemático al buscar la respuesta en un libro de autor clásico, despegándose de la inminencia de la ciencia de frontera (edge science) en juego, habilita una mirada diferente que ayuda e impulsa el desarrollo de la producción científica básicamente masculina de la época.

Este descentramiento y puesta a un lado de la forma de hacer ciencia y de pensar la ciencia por parte de las mujeres, implica una nueva mirada crítica respecto de los ideales epistemológicos de neutralidad, objetividad, racionalidad propios de un proceso histórico de producción esencialmente masculina. Siguiendo a Sandra Harding $(1986,1991)$ con su enfoque teórico feminista del punto de vista, junto con otras autoras como Hilary Rose (1983) y Dorothy Smith (1974) podemos reconocer el carácter socialmente situado de las creencias. Por lo tanto, esta situación de las mujeres podría ser entendida como una posición privilegiada en el campo epistemológico, hasta ahora dominado por hombres, en la medida en que las mujeres, debido a su posición marginal, pueden ver lo que a los hombres se les escapa desde sus posiciones de poder. Por lo tanto originarían una objetividad fuerte, un bias distinto generado por el punto de vista diferencial que superaría la tradicional objetividad, ahora considerada débil por ser parcialmente inconsciente de su postura. Ello no implicaría establecer una nueva jerarquía invertida de posturas y el consiguiente peligro de caer en otro esencialismo.

Consideramos que la presencia y actividad de la mujer en la ciencia, conlleva como resultado la producción de un conocimiento científico que logra su fortaleza por ser objetivo, pero no necesariamente neutral en lo valorativo. La presencia de otros valores, en el texto fílmico, no específicamente cognitivos sino sociales, no atenta contra la racionalidad científica, sino que por el contrario, la enriquece. En tal sentido, todos los enfoques históricos y socio-antropológicos constituyen un aporte significativo para el análisis de las representaciones de ciencia, género y poder en Talentos ocultos. 


\section{Lista de Referencias Bibliográficas}

Alic, M. [1986], (1991). El legado de Hipatia, Madrid: Siglo XXI.

Franklin, S. (1995) "The science as culture, the cultures of science" En: Annual Review of Anthropogy Volume 24.

Fischer, M. M.J. (2007) "Four Genealogies for a Recombinant Anthropology of Science and Technology” En: Cultural Anthropology. Volume 2. Issue 4. P 539-615

Foucault, M. [1976](1992). Microfísica del poder. Madrid: Las ediciones de La Piqueta

Foucault, M. [1976]2008) Historia de la sexualidad 1. La Voluntad del saber. Buenos Aires: Siglo XXI.

González García, M. I. y Pérez Sedeño, E, ( 2002). Ciencia, Tecnología y Género Número 2 / Enero - Abril

Haraway, Donna.J. (1989). Primate Visions: Gender, Race, and Nature in the World of Modern Science, Nueva York: Routledge.

Harding, S. [1986] (1995). Feminismo y ciencia, Barcelona: Morata

Harding, S. (1991). Whose Science? Whose Knowledge?, Ithaca, New York: Cornell University Press.

Jasanoff, S. (1990). The Fifth Branch: Science Advisors as Policy Markers. Cambridge. M.A.:Harvard University Press.

Martin, E. (1987). The Woman in the Body. Boston: Beacon Press

Perez Sedeño, E. (1994). “Mujeres matemáticas en la historia de la ciencia”. En Matemáticas y coeducación. OECM Ada Byron.

Rose, H. (1983), "Hand, Brain, and Heart: Towards a Feminist Epistemology for the Natural Sciences", Signs: Journal of Women in Culture and Society 9: 73-96.

Rose, H. (1994). Love, Power and Knowledge: Towards a Feminist Transformation of the Sciences, Bloomington, IN: Indiana University Press.

Sayre, A. [1975] (1997). Rosalind Franklin y el ADN, Madrid: horas y HORAS

Shetterly, M. L. (2017). Talentos Ocultos. Estados Unidos de América: Harper Collins Español

Smith, D. (1974). "Women's Perspective as a Radical Critique of Sociology", Sociological Inquiry 44.

Strathern M. (1992). Reproducing the Future: Anthropology, Kinship and the New Reproductive Technologies. Manchester, UK: Manchester University Press

Traweek, S. (1988). Life Times and Beamtimes: The Word of High Energy Physicist. M.A.: Harvard University Press.

Watson, J. [1968] (1994). La doble hélice, Barcelona: Salvat.

Abstract: The purpose of the present work is to interpret the practices and social discourses emerged from the film Hidden talents directed by Theodore Melfi (2016), from the approach to the anthropology of science on the relationship between science, gender and power.

The film, a biographical film based on the book of not fiction Hidden Figures of Margot Lee Shetterly, rescues the scientific and personal trajectories of Katherine Johnson, Dorothy Vaughn and Mary Jackson as African American Accountants of the NASA, invisible, 
disadvantaged and segregated in an era stained by the struggle for the recognition of civil rights and social inclusion. The film revives and explores the human drama expressed by its double condition of women and people of African descent in a predominantly male scientific community interested in reversing the Soviet advance in the domain of space and develop competitively the space race in the United States.

The material provided by the film, very consistent with the book on which it is based, allows you to analyze not only the role of the woman as a maker of science, but go further in understanding their practices and scientific representations, analyzing their advances and limits in the production of knowledge, its specific features provided in the contents obtained and the type of knowledge located socially. In this sense, the anthropology of science, especially his from pioneers in the field of feminist and cultural studies, provides a specific background of theoretical treatment accorded to a large extent although not unanimously innovative concepts contributed by Foucault (1976) on the knowledge buried by a resurgent held by a few science.

Keywords: Science - gender - power - culture - anthropology

Resumo: O propósito deste trabalho é interpretar as práticas e discursos sociais decorrentes do filme Talentos ocultos dirigido por Theodore Melfi (2016), desde a perspectiva da antropologia da ciência enquanto à relação entre ciência, gênero e poder.

O filme, uma longametragem biográfica baseada no livro de não ficção Hidden Figures de Margot Lee Shettersly, resgata as trajetórias pessoais e científicas de Katherine Johnson, Dorothy Vaughn e Mary Jackson como calculistas afroamericanas da NASA, invisibilizadas, desfavorecidas e segregadas numa época marcada pela luta pelo reconhecimento dos direitos civis e a inclusão social. O filme reaviva e explora o drama humano expresso pela sua dupla condição de mulheres e afrodescendentes numa comunidade científica preponderantemente masculina interessada em reverter o avanço soviético no domínio do espaço e desenvolver competitivamente a carreira espacial dos Estados Unidos.

A matéria oferecida pelo filme, muito concordante com o livro no qual se baseia, permite analisar não só o papel da mulher como sujeito fazedor de ciência mas também ir além na compreensão de suas práticas e representações científicas, analisando os seus avanços e limites na produção do conhecimento, as suas especificidades e contribuições aos conteúdos obtidos e o tipo de saberes socialmente situados. Nesse sentido, a antropologia da ciência, especialmente no trabalho de suas pioneiras, vindas do campo dos estudos feministas e culturais, oferece um conteúdo específico de tratamento teórico que reconhece em grande parte, embora não unanimemente, os novos conceitos oferecidos por Foucault (1976) sobre o ressurgimento dos saberes escondidos por uma ciência possuída por uns poucos.

Palavras chave: Ciência - gênero - poder - cultura - antropologia.

[Las traducciones de los abstracts fueron supervisadas por el autor de cada artículo] 